\title{
A study on a mathematical model of gas in accumulator using van der Waals equation
}

\author{
Shuto Miyashita*, Shuce Zhang**, and Kazushi Sanada*** \\ * Department of Mechanical and Control Engineering, Graduate School of Science and Engineering \\ Tokyo Institute of Technology, Ookayama, Tokyo, Japan \\ ** Department of Systems Integration, Graduate School of Engineering \\ Yokohama National University, Yokohama City, Japan \\ *** Faculty of Engineering, Yokohama National University, Yokohama City, Japan \\ E-mail: sanada-kazushi-sn@ynu.ac.jp
}

\begin{abstract}
As mathematical models of hydraulic accumulator, thermodynamics, heat transfer, and gas models are briefly described, including the isothermal and the adiabatic equations of the van der Waals equation. Measurement of gas behavior using a bladder type accumulator and a piston type accumulator was carried out. Measured data of a discharge test of the bladder accumulator and a charge test of the piston type accumulator were used to validate the models.
\end{abstract}

Keywords: Accumulator, Gas model, Van der Waals equation, Oil-hydraulics

\section{Introduction}

An accumulator is an important component for oil-hydraulic circuits. Because of complexity of gas behavior in the accumulator, it is not easy to do accurate prediction of the accumulator performance. There have been many research papers on modeling the gas behavior of the accumulator. Otis introduced a concept of the thermal time constant representing heat transfer effect [1][2][3].

In addition to the heat transfer effect and thermodynamics, a real gas model is needed to predict the accumulator performance. Beattie-Bridgeman equation [3] and SoaveRedlich-Kwong equation [4] are typical mathematical models. The van der Waals equation is a basis of these real gas models. It is easy to treat the van der Waals equation mathematically. For examples, the adiabatic equation and the isothermal equation are established as simple mathematical equations.

In this study, an experiment of a bladder type accumulator and a piston type accumulator was performed. These accumulators were connected to each other. Discharge process and charge process of the accumulators were measured simultaneously. The measured data were used to validate the adiabatic and the isothermal equation of the van der Waals equation.

In the section 2, a mathematical model of accumulator using thermodynamics and heat transfer are briefly introduced. Ideal gas model and real gas models are described in the section 3. Measurement of a bladder type accumulator and a piston type accumulator is explained in the section 4 . In the section 5 , the adiabatic equation and the isothermal equation of the van der Waals equation are validated with the measured data. Finally, results are summarized in the section 6.

\section{Accumulator model}

In a mathematical model of gas-charged accumulator, thermodynamics, heat transfer and a gas model are considered. In this section, the thermodynamics and the heat transfer are briefly introduced[3].

Energy balance of accumulator is shown in Figure 1. Nitrogen gas of mass, $m_{\text {gas }}$, has internal energy of $u$. The nitrogen gas receives thermal energy $Q$ from the surroundings and performs work $W$ to the surroundings. Mathematical modelling begins by the conservation of energy equation:

$$
m_{g a s} \frac{d u}{d t}=\frac{d Q}{d t}-\frac{d W}{d t}
$$

Heat transfer between gas and the surroundings is represented by:

$$
\frac{d Q}{d t}=h A\left(T_{w}-T\right)
$$

Rate of work output is: 


$$
\frac{d W}{d t}=P \frac{d V}{d t}
$$

Change of internal energy of gas is represented by:

$$
d u=c_{v} d T+\left[T\left(\frac{\partial P}{\partial T}\right)_{v}-P\right] d v
$$

Substituting Eq.(2), Eq. (3), and Eq.(4) to Eq.(1) yields:

$$
\tau \frac{d T}{d t}+T=T_{w}-\frac{T \tau}{c_{v}}\left(\frac{\partial P}{\partial T}\right)_{v} \frac{d v}{d t} .
$$

The parameter $\tau$ is called the thermal time constant:

$$
\tau=\frac{m_{g a s} c_{v}}{h A} .
$$

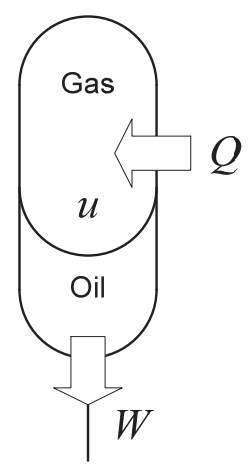

Figure 1: Energy balance of accumulator

The concept of thermal time constant, Eq.(6), represents heat transfer between gas and the surroundings through rubber bag and vessel wall. Figure 2 is a plot of charged-gas pressure. A quick compression process from $\mathrm{A}$ to $\mathrm{B}$ increases the gas pressure. Due to the quick compression, gas temperature is increased. Then thermal energy of gas will be transferred to the surroundings through the rubber bag and the wall of the accumulator. Due to the heat transfer, gas pressure decreases gradually as shown in Figure 2. The thermal time constant $\tau$ is the time it takes for the gas pressure to drop by 63.2 percent.

\section{Mathematical models of gas in accumulator}

As shown in Figure 3, during the charge process from A to B and the discharge process from $\mathrm{C}$ to $\mathrm{D}$, gas volume changes between $V_{1}$ and $V_{2}$. The PV diagram is shown in Figure 4. The process from A to $\mathrm{B}$ is the charge process. Gas volume decreases from $V_{1}$ to $V_{2}$. The process from $\mathrm{C}$ to $\mathrm{D}$ is the discharge process. Gas volume increases from $V_{2}$ to $V_{1}$. Gas pressure changes depending on the volume and the state change of gas. Models of state change of gas are ideal gas model and real gas model.

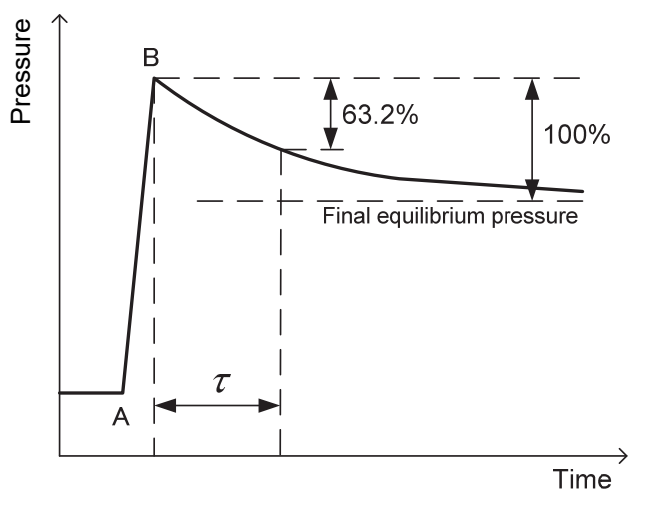

Figure 2 Thermal time constant [3]

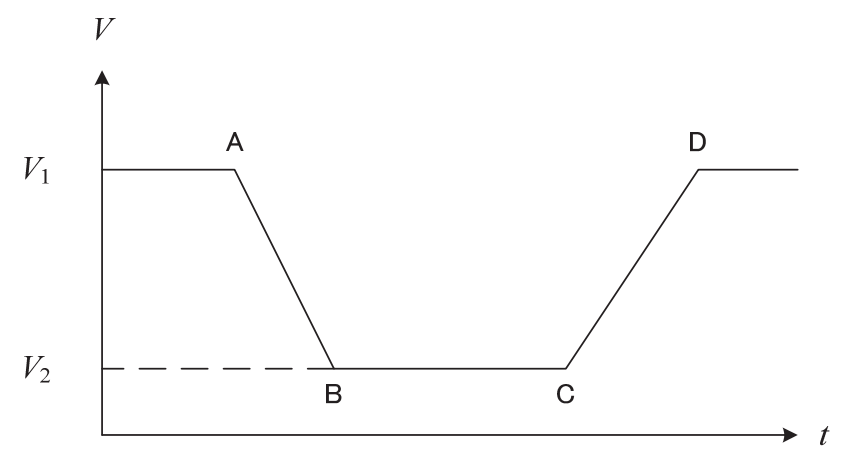

Figure 3 Volume change of gas in the accumulator

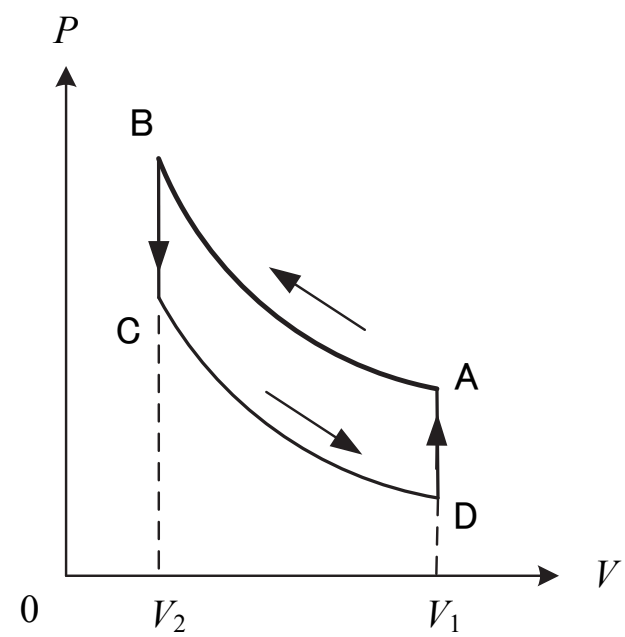

Figure 4 P-V diagram of accumulator gas

\subsection{Ideal gas model}

The ideal gas model is a virtual model in which volume of gas molecule and intermolecular force are ignored. Ideal gas state equation is

$$
P V=m R T \text {. }
$$

This equation represents the relationship of state variables, such as pressure, volume, and temperature. 
For isothermal change of ideal gas, the relationship between absolute pressure $P$ and volume $V$ is represented by:

$$
P V=\text { Const., }
$$

which is known as the Boyle's law.

For adiabatic change of ideal gas,

$$
P V^{\kappa}=\text { Const., }
$$

where $\kappa$ is a specific-heat ratio.

For the case in which the state of ideal gas changes in between isothermal and adiabatic conditions, the polytropic change of state is used:

$$
P V^{n}=\text { Const., }
$$

where $n$ is the polytropic exponent.

\subsection{Real gas models}

It is well known that every material has three phases, such as solid, liquid, and gaseous phases as shown in Figure 5. The state of material is determined by temperature and pressure. By the change of temperature and pressure, the state of material may change. A boiling point is the temperature at which liquid state changes to gaseous state. The higher the pressure, the higher the boiling point. However, the increase of the boiling point is limited. The maximum limit of the boiling point is the critical point. When material exits under temperature and pressure beyond the critical point, the state is called "super critical fluid". The super critical fluid has both gaseous and liquid characteristics. In Table 1, critical properties of nitrogen gas are listed. These critical properties are used for real gas models.

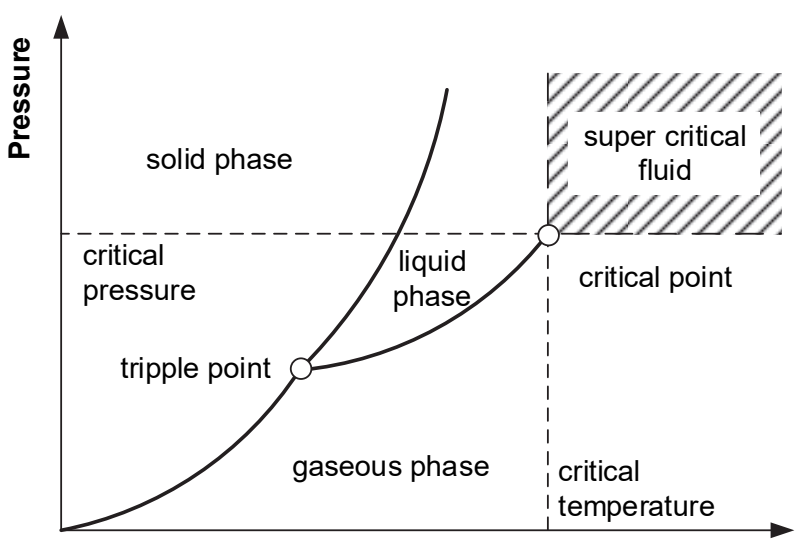

Temperature

Figure 5 Phase diagram and super critical fluid

Table 1 Critical properties of $\mathrm{N}_{2}$ gas

\begin{tabular}{|l|c|l|}
\hline Critical pressure & $P_{c}$ & $3.394388 \mathrm{MPa}$, abs. \\
\hline Critical specific volume & $v_{c}$ & $9.003 \times 10^{-5} \mathrm{~m}^{3} / \mathrm{mol}$ \\
\hline Critical temperature & $T_{c}$ & $126.1 \mathrm{~K}$ \\
\hline Gas constant & $R$ & $8.314501 \mathrm{~J} /(\mathrm{Kmol})$ \\
\hline
\end{tabular}

The van der Waals equation is a basic equation of real gas model:

$$
\left(P+\frac{a m^{2}}{V^{2}}\right)(V-m b)=m R T .
$$

The parameters $a$ and $b$ are calculated from the critical properties:

$$
\begin{gathered}
a=\frac{9}{8} R v_{c} T_{c}, \\
b=\frac{1}{3} v_{c} .
\end{gathered}
$$

Assuming constant temperature, the isothermal equation is obtained:

$$
\left(P+\frac{a m^{2}}{V^{2}}\right)(V-m b)=\text { const. }
$$

The equation of state for adiabatic process of the van der Waals equation of state is

$$
\left(P+\frac{a m^{2}}{V^{2}}\right)(V-m b)^{H}=\text { const. }
$$

where the adiabatic exponent $H$ is

$$
H=\frac{R}{c_{v}}+1 .
$$

The isothermal equation of the van der Waals equation, Eq.(14), and the adiabatic equation of the van der Waals equation, Eq.(15), correspond to those of ideal gas, Eq.(8) and Eq.(9), respectively. The adiabatic exponent $H$ of Eq.(16) of the van der Waals equation corresponds to the specific-heat ratio $\kappa$ of Eq.(9) of ideal gas. For real gas, the adiabatic exponent $H$ is not identical to the specific-heat ratio $\kappa$. In ideal gas case, because of the Mayer law, $c_{p}-c_{v}=R$, the adiabatic exponent $H$ is equal to the specific-heat ratio $\kappa=$ $c_{p} / c_{v}$.

A more accurate real gas model is the Beattie-Bridgeman model [1][2]. The Beattie-Bridgeman equation is more complex:

$$
P=\frac{R T}{v}+\frac{\beta}{v^{2}}+\frac{\gamma}{v^{3}}+\frac{\delta}{v^{4}} .
$$

Pourmovahed [3] used the Benedict-Webb-Rubin equation which is much more complex: 


$$
\begin{aligned}
P=\frac{R T}{v}+\frac{\left(B_{0} R T\right.}{\left.-A_{0}-\frac{C_{0}}{T^{2}}\right)}+\frac{(b R T-a)}{v^{3}} \\
+\frac{a \alpha}{v^{6}} \\
+\frac{c\left(1+\frac{\gamma}{v^{2}}\right) e^{-\gamma / v^{2}}}{v^{3} T^{2}}
\end{aligned}
$$

A commercial software AMESim [5] uses the Soave-RedlichKwong model [4]:

$$
\begin{aligned}
& \left(P+\frac{a \alpha(T)}{v(v+b)}\right)(v-b)=R T . \\
& a=\frac{0.4278 R^{2} T_{c}^{2}}{P_{c}} \\
& b=\frac{0.0867 R T_{c}}{P_{c}} \\
& \alpha(T)=\left[1+m\left(1-\sqrt{1-\frac{T}{T_{c}}}\right)\right]^{2}
\end{aligned}
$$

Hansen and Rasmussen [6] carried out experiments using a piston accumulator for wind turbines under a wide range of temperature. They compared the Benedict-Webb-Rubin Equation and the Soave-Redlich-Kwong equation with the experiments and concluded that the Soave-Redlich-Kwong equation is more efficient and accurate enough for wind turbine applications.

It is known that the van der Waals equation has limited accuracy. Improved real gas models have been used, such as the Soave-Redlich-Kwong equation. However, the van der Waals equation is easy to treat mathematically. For examples, the adiabatic equation and the isothermal equation can be derived from the van der Waals equation, as introduced by Eq.(14) and Eq.(15).

\section{Measurement of gas behavior}

In this study, experiments were carried out to measure gas behavior during charge and discharge processes using a bladder type accumulator and a piston type accumulator. An experimental circuit is shown in Figure 6. The bladder type accumulator (a) and the piston type accumulator (b) were connected by a valve (e). The oil-hydraulic pump (f) with the relief valve (k) supplied pressurized oil to one of the accumulators. By opening the stop valve $(\mathrm{g})$ and closing the valves (e), (h), (i) and (j), the pump prefilled the bladder type accumulator (a). The condition was kept for seven hours to reach steady state condition of temperature of the accumulators. All windows in the laboratory were covered by black cardboards to keep the room temperature. Temperature of the room was regulated by air condition. By opening the valve (j), the piston type accumulator (b) was fully extracted.
Closing the valve (g) and (j), and opening the valve (e), the bladder type accumulator was discharged and the piston type accumulator was charged. The piston displacement was measured by a potentiometer (d) and gas volume in the piston type accumulator was calculated. Gas volume in the bladder type accumulator was calculated based on the volume of oil charged in the piston type accumulator. The piston displacement, the surrounding temperature, and pressure of gas in both accumulators were recorded.

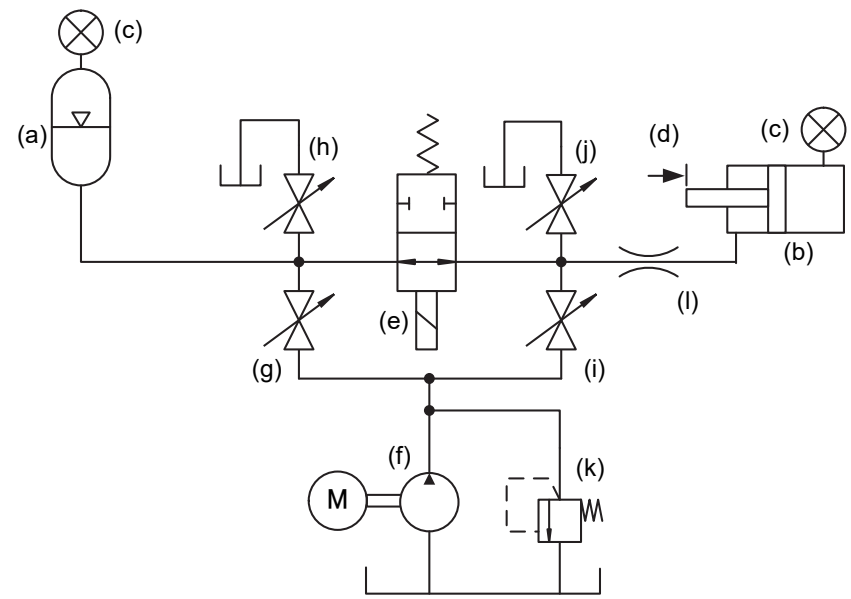

Figure 6 An experimental circuit using a bladder type and a piston type accumulators

\section{Considerations}

As an example of the measurements, a PV-diagram of a discharged test of the bladder type accumulator is shown in Figure 7. The discharge process was carried out by 10 seconds and the surrounding temperature was $301 \mathrm{~K}$. The black solid line is the measured results. The point $\mathrm{A}$ is the initial condition of $34.5 \mathrm{MPa}$, abs. and $2.65 \times 10^{-3} \mathrm{~m}^{3}(2.65 \mathrm{~L})$. By discharging, the gas volume increased to the point $\mathrm{B}$ and the gas pressure decreased. A broken line is calculated results of Eq.(15), the adiabatic equation of the van der Waals equation (indicated by "vdW(adiabatic)"). A dotted line is calculated results of Eq.(14), the isothermal equation of the van der Waals equation (indicated by "vdW(isothermal)"). In this particular measurement, the discharge process of 10 seconds showed similar behavior as the adiabatic equation of the van der Waals equation.

Measured results of a charge test of the piston type accumulator are plotted in Figure 8. The measurement was performed simultaneously as the data shown in Figure 7 . The point $\mathrm{A}$ is the initial condition of $4.4 \mathrm{MPa}$, abs. and $7.4 \times 10^{-3}$ $\mathrm{m}^{3}(7.4 \mathrm{~L})$. The discharge process was carried out by 10 seconds and the surrounding temperature was $301 \mathrm{~K}$. The solid line is the measured results. By charging, the gas volume decreased and the gas pressure increased. The point B is the end of charge process. After the charge process, beyond the point $\mathrm{B}$, the gas pressure decreased by about $0.6 \mathrm{MPa}$. It can be estimated that it was due to the heat transfer effect between the gas and the surroundings. The broken line and the dotted line are calculated results of the adiabatic equation of Eq.(15) and the isothermal equation of Eq.(14) of the van der Waals 
equation. The measured results almost follow the adiabatic line, but there can be seen a certain degree of discrepancy from the adiabatic equation. As described in the reference [6], in the piston accumulator, thermal energy of gas can transfer directly through the accumulator wall to the surroundings. The adiabatic condition is not easy to establish. Heat conductive phenomenon inside the rubber membrane and the steel wall of accumulator is needed to investigate.

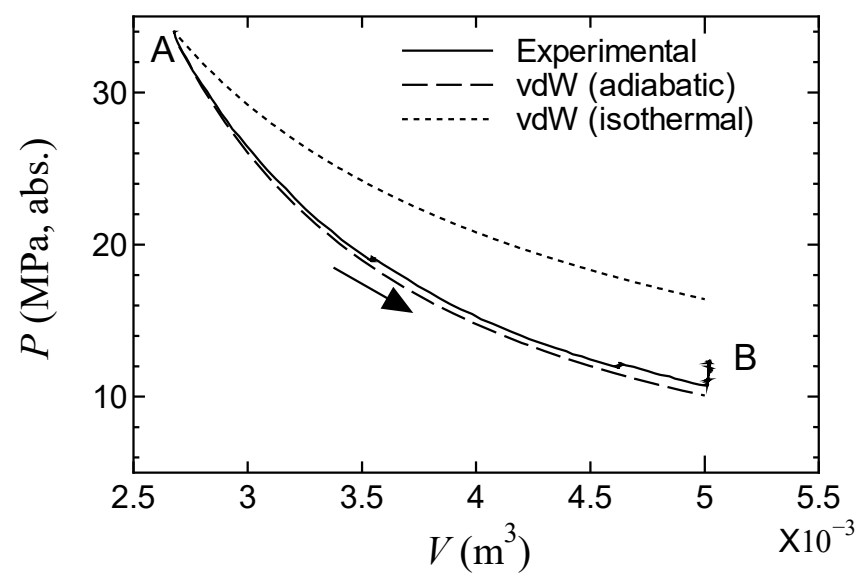

Figure 7 Measured results of discharge test of the bladder type accumulator and calculated results of the van der Waals equations $(10$ s, 301K)

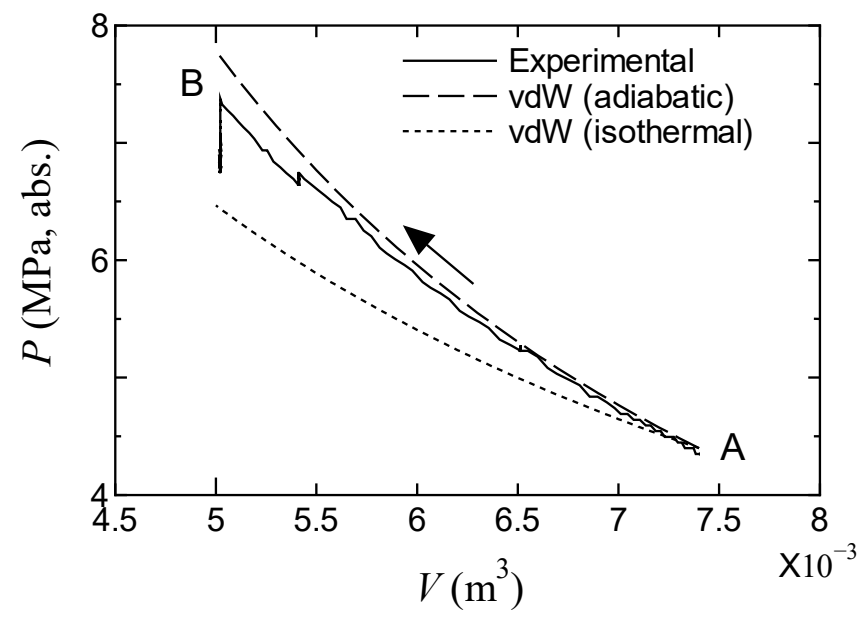

Figure 8 Measured results of charge test of the piston type accumulator and calculated results of the van der Waals equations $(10 \mathrm{~s}, 301 \mathrm{~K})$

\section{Conclusions}

The van der Waals equation is a basis of real gas model. The isothermal and the adiabatic equation are established as a simple mathematical equation. In this study, measurements were performed using a bladder type accumulator and a piston type accumulator. These accumulators were connected to each other. Discharge and charge processes were measured simultaneously. The measured data were compared with the adiabatic equation and the isothermal equation of the van der Waals equation. The test processes of 10 seconds were almost approximated by the adiabatic equation of van der Waals equation. Other experiments of process time of longer than
10 seconds have been conducted. It is a future work to investigate these measured data by comparison of real gas models, such as the van der Waals equation and the SoaveRedlich-Kwong equation.

\section{Nomenclatures}

\begin{tabular}{cll}
\hline Designation & Denotation & $\mathrm{Unit}$ \\
\hline$A$ & surface area of wall & $\mathrm{m}^{2}$ \\
$c_{p}$ & constant pressure specific heat & $\mathrm{J} /(\mathrm{kgK})$ \\
$c_{v}$ & constant volume specific heat & $\mathrm{J} /(\mathrm{kgK})$ \\
$h$ & heat transfer coefficient & $\mathrm{W} /\left(\mathrm{m}^{2} \mathrm{~K}\right)$ \\
$m$ & number of moles & - \\
$m_{g a s}$ & mass of gas & $\mathrm{kg}$ \\
$n$ & polytropic exponent & - \\
$P$ & absolute pressure of gas & $\mathrm{Pa}, \mathrm{abs}$. \\
$Q$ & heat energy & $\mathrm{J}$ \\
$R$ & gas constant & $\mathrm{J} /(\mathrm{kgK})$ \\
$T$ & gas temperature & $\mathrm{K}$ \\
$T_{w}$ & wall temperature & $\mathrm{K}$ \\
$t$ & time & $\mathrm{s}$ \\
$u$ & internal energy & $\mathrm{J}$ \\
$v$ & specific volume & $\mathrm{m}{ }^{3} / \mathrm{kg}$ \\
$V$ & gas volume & $\mathrm{m}$ \\
$\kappa$ & specific-heat ratio & thermal time constant \\
$\tau$ & &
\end{tabular}

\section{Acknowledgements}

Professor Kitagawa and Professor Kagawa (Tokyo Institute of Technology) gave constructive comments and warm encouragement. Nippon Accumulator Co., Ltd. supported the experiments.

\section{References}

[1] D. R. Otis, New developments in predicting and modifying performance of hydraulic accumulators, National Conference on Fluid Power: 473-483, 1974.

[2] F. T. Elder, D. R. Otis, Accumulators: The role of heat transfer in fluid power losses, 4th International Fluid Power Symposium: D2-27-D2-37, 1975.

[3] A. Pourmovahed, D. R. Otis, An experimental thermal time-constant correlation for hydraulic accumulator, Transactions of the ASME, Journal of Dynamic Systems, Measurement, and Control, Vol.112: 117-121, March 1990. 
[4] G. Soave, Equilibrium constants from a modified Redlich-Kwong equation of state, Chemical Engineering Science, Vol.27, pp.1197-1203, 1972.

[5] LMS Amehelp, HA0021 - hydraulic accumulator with heat exchange (semi-perfect or real gas behavior), 2010.

[6] H. B. Hansen, P. W. Rasmussen, Modeling Hydraulic Accumulators for use in Wind Turbines, The 13th Scandinavian International Conference on Fluid Power, SICFP2013, p.327-324, 2013. 